\title{
Multicore optical fibres for next generation telecommunication transmission systems and components
}

\author{
Marek Napierała, ${ }^{* 1,2}$ Anna Ziołowicz ${ }^{1,3}$, Anna Pytel ${ }^{1}$, Łukasz Szostkiewicz ${ }^{4}$, Michał Murawski ${ }^{4}$, \\ Tadeusz Tenderenda ${ }^{1}$, Łukasz Ostrowski ${ }^{1}$, Michał Szymański ${ }^{4}$, Michalina Jóźwik ${ }^{1}$, Marta Filipowicz ${ }^{1}$, \\ Mariusz Makara ${ }^{1}$, Krzysztof Poturaj $^{5}$, Paweł Mergo ${ }^{5}$, and Tomasz Nasiłowski ${ }^{1}$ \\ ${ }^{1}$ InPhoTech,Ltd., Slominskiego 17/31, 00-195 Warsaw \\ ${ }^{2}$ Faculty of Advanced Technologies and Chemistry, Military University of Technology, \\ Kaliskiego 2, 00-908 Warsaw \\ ${ }^{3}$ Faculty of Physics, Warsaw University of Technology, Koszykowa 75, 00-662 Warsaw \\ ${ }^{4}$ Polish Centre For Photonics and Fibre Optics, Rogoznica 312, 36-060 Glogow Malopolski \\ ${ }^{5}$ Laboratory of Optical Fibre Technology, Faculty of Chemistry, Maria Curie-Skłodowska University, \\ Pl. Maria Curie-Sklodowska 3, 20-031 Lublin
}

Received August 28, 2015; accepted September 23, 2015; published September 30, 2015

\begin{abstract}
We report on a new type of multicore fibres dedicated to a new generation of telecommunication networks. We designed and fabricated seven core fibres for (i) passive signal transmission, (ii) signal amplification and (iii) $1 \times 7$ signal division. In each case, the same cores and air-holes arrangement is used. However, a different operation is achieved by employing different cores doping and varying structural parameters of fibre, such as air-holes and cores diameters. We present the experimental results of measurements performed for developed multicore fibres and multicore fibre components.
\end{abstract}

Space division multiplexing (SDM) is recognized as a way of facing up a demand for ultra-high capacity transmission systems [1]. SDM may rely on multicore fibres (MCFs), in which each core constitutes an independent channel or on few mode fibres. In the latter case, mode division multiplexing (MDM) is used, which means that each mode of the fibre is an independent transmission channel. Both techniques can be also used simultaneously. In fact, the few-mode MCFs have recently been employed to beat transmission capacity records (over 255Tb/s [2] and 1Pb/s [3]). While this approach is undoubtedly remarkable, the downsides of a few-mode transmission (e.g. incompatibility with existing single mode transmission standards, not matured mode addressing techniques, etc.) need also to be taken into account.

In the future telecommunication systems based on few mode MCFs, change of transceiver technology and whole telecom infrastructure will be needed. However, from the commercial point of view, that kind of change cannot take place at once. For this reason, the system based on multicore fibre with single-mode cores seems to be the proper buffer solution before spreading the MDM technology.

Although the research on MCFs is well advanced and several high bit rate transmissions have already been shown [2-4], the commercial transmission line based on

*E-mail: mnapierala@inphotech.pl
MCFs remains still a challenge, because of compatibility issues with the existing networks.

In the paper, we report on an MCF, which is easily adaptable to existing networks. This feature is obtained by optimizing the transmission parameters of our fibre to be in line with the actual telecommunication standards and recommendations in terms of single-mode operation, mode field diameter (MFD), low losses (transmission and bending), dispersion characteristics and outer diameter. As a baseline we chose International Telecommunication Union recommendation G.652 ("Characteristics of a single-mode optical fibre and cable"). In terms of macrobending loss we used recommendation G.657 ("Characteristics of a bending-loss insensitive singlemode optical fibre and cable for the access network") as a reference. Another goal, of our MCF design was to suppress crosstalk (XT) between cores. In order to decrease XT, many approaches have been presented. The reduction of XT requires an application of core isolation, which can be obtained by: differentiating cores diameters or/and their doping level (i.e. by creating so called heterogeneous cores) [5-6], applying the areas of decreased refractive index around the cores (by creating trench [4, 7] or hole-assisted [8, 9] fibres) or by combining the listed methods. It is worth mentioning that there are no recommendations or norms in terms of XT, which makes the comparison of different fibres difficult.

To provide high core density and assure a negligible XT, we developed the MCF with a hole-assisted structure (Fig. 1) formed out of seven basic cells. The basic cell consists of one core made out of silica glass doped with $\mathrm{GeO}_{2}$ surrounded by twelve air-holes. Air-holes and cores are arranged on a hexagonal lattice, which enables manufacturing such MCF by means of a stack-and-draw method.

The main function of air-holes is to decrease XT between cores. In addition, the hole-assisted structure makes the structure bend-insensitive; this approach is 
known for single core fibres [10]. On the other hand, the arrangement and size of air holes need to be precisely designed and controlled to avoid affecting the propagation characteristics of doped cores and prevent the propagation of higher order modes in the cladding [11].

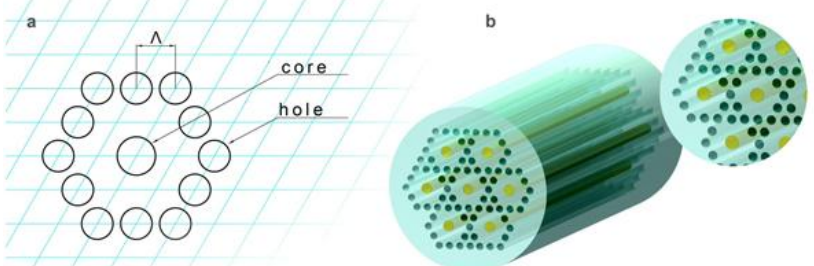

Fig. 1. (a) Basic cell of proposed hole-assisted MCF with marked lattice constant $\Lambda$. (b) Composition of seven basic cells with three additional holes playing the role of markers.

We manufactured two series of such MCF, which are presented in Fig. 2.
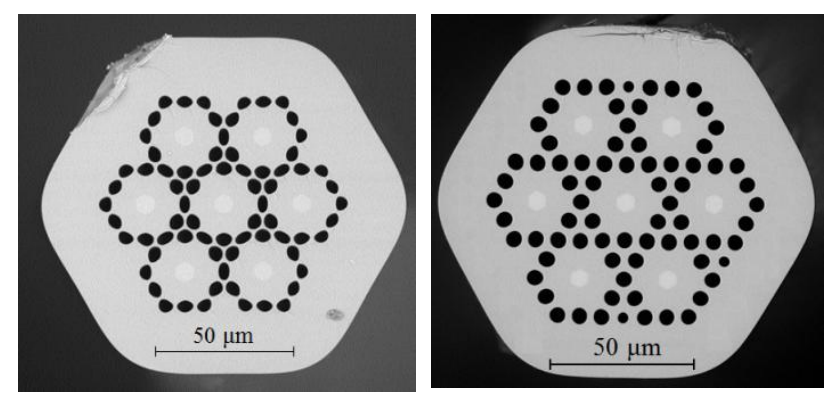

Fig. 2. SEM images of cross sections of manufactured MCFs: fibre A with strong cores isolation (left side), fibre B with lesser cores isolation (right side).

We examined the manufactured fibres paying special attention to their transmission and bending loss as well as dispersion characteristics. The parameters measured for fibre B are presented in Tab. 1.

Tab. 1 Geometrical parameters for fibres A and B and transmission parameters of fibre B.

\begin{tabular}{|c|c|c|c|}
\hline & \multirow{2}{*}{ Parameter } & \multicolumn{2}{|c|}{ Value } \\
\hline & & Fiber A & Fiber B \\
\hline \multirow{4}{*}{ 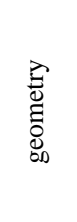 } & core diameter & $(7.4 \pm 0.1) \mu \mathrm{m}$ & $(6.2 \pm 0.1) \mu \mathrm{m}$ \\
\hline & air-hole diameter & $(5.9 \pm 0.1) \mu \mathrm{m}$ & $(5.6 \pm 0.1) \mu \mathrm{m}$ \\
\hline & cladding diameter & $(120.8 \div 131.2) \mu \mathrm{m}$ & $(121.4 \div 130.3) \mu \mathrm{m}$ \\
\hline & lattice constant & $(7.1 \pm 0.1) \mu \mathrm{m}$ & $(7.6 \pm 0.1) \mu \mathrm{m}$ \\
\hline \multirow{4}{*}{ 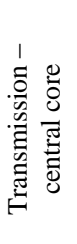 } & $\begin{array}{l}\text { transmission loss } \\
\text { @ 1550nm }\end{array}$ & $\sim 25 \mathrm{~dB} / \mathrm{km}$ & $\sim 1.5 \mathrm{~dB} / \mathrm{km}$ \\
\hline & ZDW & $1314 \mathrm{~nm}$ & $1315.2 \mathrm{~nm}$ \\
\hline & D@1550nm & $18.4 \mathrm{ps} /(\mathrm{nm} \cdot \mathrm{km})$ & $14.5 \mathrm{ps} /(\mathrm{nm} \cdot \mathrm{km})$ \\
\hline & slope @ ZDW & $0.03 \mathrm{ps}^{2} /(\mathrm{nm} \cdot \mathrm{km})$ & $0.06 \mathrm{ps}^{2} /(\mathrm{nm} \cdot \mathrm{km})$ \\
\hline
\end{tabular}

For the outer cores of fiber $\mathrm{B}$, the transmission loss is at the level of several $\mathrm{dB} / \mathrm{km}$. This relative big transmission loss results mainly from the manufacturing imperfections (laboratory scale of production), and should be substantially reduced for an optimized fabrication process. There are no physical obstacles to achieve final transmission loss below $0.2 \mathrm{~dB} / \mathrm{km}$, when the fiber is drawn in industrial conditions.

The dispersion characteristics for outer cores (three of six were under tests) remain in the ranges:

- ZDW: $(1299.1 \div 1320.5) \mathrm{nm}$,

- D @ $1550 \mathrm{nm:}(15,04 \div 15,24) \mathrm{ps} /(\mathrm{nm} \cdot \mathrm{km})$,

- $\quad$ slope @ ZDW: $(0.050 \div 0.094) \mathrm{ps}^{2} /(\mathrm{nm} \cdot \mathrm{km})$.

In some cases, the obtained values do not fulfil G.652 recommendation, but the differences were on the margin of error. For this structure, the numerical aperture and mode field diameter are equal to 0.13 and $9 \mu \mathrm{m}$, respectively.

Bending loss was measured for all cores by winding the fibre on mandrels with radii: $15 \mathrm{~mm}, 10 \mathrm{~mm}, 7.5 \mathrm{~mm}$ and $5 \mathrm{~mm}$. For each mandrel we tested the number of turns from one to ten and we checked the difference in the transmitted power in relation to a straight sample. For every combination of mandrel radius and number of turns, the loss does not exceeded $0.05 \mathrm{~dB}$ at $1550 \mathrm{~nm}$. Thus, the fibre satisfies G.657.B3 recommendation. In addition, the splice loss between one core of fibre B and standard SMF-28 is below $0.5 \mathrm{~dB}$, which shows very high consistency of mode fields of these fibres.

The same arrangement of basic cells, as in the transmission MCF, was also used for the active MCF (Fig. 3), but in this case the cores and holes diameters are decreased. The cores are doped with erbium ions, which enables the amplification of a signal within the telecommunication C-band.

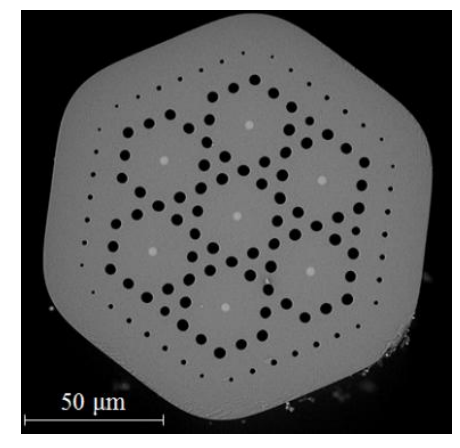

Fig. 3. SEM image of cross-section of the active MCF.

Numerical simulations unveiled that besides the fundamental mode, there is one higher order mode, which propagates in the basic cell. However, the loss for this higher order mode is big, i.e. over $7 \mathrm{~dB} / \mathrm{m}$ and over $2 \mathrm{~dB} / \mathrm{m}$ at wavelengths of $980 \mathrm{~nm}$ and $1550 \mathrm{~nm}$, respectively. Thus, it should not affect the amplification characteristics 
of the fibre. The achieved gain for this fibre is approx. $21 \mathrm{~dB}$ at a wavelength of $1550 \mathrm{~nm}$ (signal power of $-9 \mathrm{dBm}$ and pump power of $150 \mathrm{~mW}$ ).

As it was mentioned, the air-hole diameter has crucial influence on the XT. By decreasing the air-hole diameters, one can obtain the fibre with coupled cores, which means that optical power is transferred between cores. We used this phenomenon to develop an optical fibre power splitter. In our experiment, we applied power division into cores of a seven-core MCF. The SMF-28 fibre was used to introduce light into a central core of the MCF. The division of power in such an element is presented in Fig. 4.

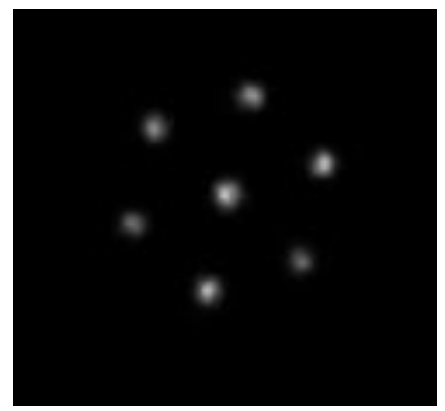

Fig. 4. Camera view on power division among all cores of the fibre A.

The best parameters obtained for this kind of $1 \times 7$ splitter for fibre A are the loss of $1.7 \mathrm{~dB}$ and the power distribution uniformity (defined as the power difference between the average power per core and the extreme power level in the core at the output of the splitter) of 1 $\mathrm{dB}$.

The presented all-fibre splitter design, in comparison to the fused biconical taper (FBT) technology, allows for realization of power division a into higher number of output fibres. The splitter satisfies also other requirements regarding optical fibre splitters, such as easy and durable construction, resistance to the change of external conditions and low price.

In summary, we have demonstrated the research on seven-core MCFs. In particular, the parameters of passive transmission fibre were optimized with a view to adapting them to actual standards and recommendations. The parameters of the developed passive MCF are in a very good agreement with G.652 and G.657 recommendations, thus the fibre is compatible with the existing networks and can be readily applied. The use of such fibre allows for a sevenfold transmission capacity increase.

We have also demonstrated the amplification of a signal with the use of an amplifier based on active MCF and we achieved the gain of approx. $21 \mathrm{~dB}$. In addition, the reduction of isolation between the cores of MCF allowed us to fabricate a $1 \times 7$ splitter with a loss of $1.7 \mathrm{~dB}$ and power division uniformity of $1 \mathrm{~dB}$. The presented MCFs and MCF-based components will allow for the transition from the existing transmission networks based on single core fibres to future networks based on MCFs.

This research was partially supported by the Polish National Science Centre within the project 2013/09/D/ST7/03961 as well as by the National Centre for Research and Development within the research projects PBS1/B3/12/2012 and POIG.01.03.01-06-085/12 and Ministry of Science and Higher Education within the project DI2013 019343. This research project has been also supported by the European Commission under the 7th Framework Programme within the project BEACON FP7-Space-2013-1-607401.

\section{References}

[1] D.J. Richardson, J.M. Fini, L.E. Nelson, Nat. Photonics 7, 354 (2013), http://doi.org/10.1038/nphoton.2013.94

[2] R.G.H. van Uden et al., Nat. Photonics 8, 865 (2014), http://doi.org/10.1038/nphoton.2014.243

[3] H. Takara et al., ECOC Tech. Dig. 3, paper Th.3.C.1. (2012), http://doi.org/10.1364/ECEOC.2012.Th.3.C.1

[4] H. Takara et al., Opt. Expr. 20, 10100 (2012), http://doi.org/10.1364/OE.20.010100

[5] M. Koshiba, K. Saitoh, Y. Kokubun, IEICE Electron. Expr. 6, 98 (2009), http://doi.org/10.1587/elex.6.98

[6] Y. Kokubun, T. Watanabe, 17th Microoptics Conference, Sendai, (2011),

http://ieeexplore.ieee.org/xpl/articleDetails.jsp?arnumber=6110376

[7] J. Tu et al., J. Light. Technol. 31, 2590 (2013) http://doi.org/10.1109/JLT.2013.2271514

[8] B. Yao et al., Optical Fiber Communication Conference and Exposition (OFC/NFOEC) OFC, Los Angeles (2012), http://doi.org/10.1364/OFC.2012.OM2D.5

[9] K. Imamura et al., 34th European Conference on Optical Communication (2008), http://doi.org/10.1109/ECOC.2008.4729437

[10] K. Saitoh, Y. Tsuchida, M. Koshiba, Opt. Lett. 30, 1779 (2005), http://doi.org/10.1364/OL.30.001779

[11] A. Ziolowicz et al., Appl. Phys. Lett. 105, 081106 (2014), http://doi.org/10.1063/1.4894178 